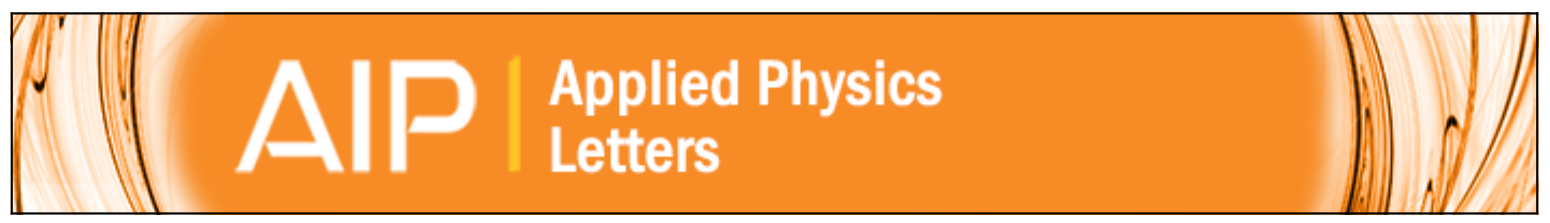

\title{
A method to measure the electron temperature and density of a laser-produced plasma by Raman scattering
}

H. Jang, M. S. Hur, J. M. Lee, M. H. Cho, W. Namkung, and H. Suk

Citation: Applied Physics Letters 93, 071506 (2008); doi: 10.1063/1.2973395

View online: http://dx.doi.org/10.1063/1.2973395

View Table of Contents: http://scitation.aip.org/content/aip/journal/apl/93/7?ver=pdfcov

Published by the AIP Publishing

\section{Articles you may be interested in}

Simultaneous measurement of electron and heavy particle temperatures in He laser-induced plasma by Thomson and Rayleigh scattering

Appl. Phys. Lett. 102, 134108 (2013); 10.1063/1.4801467

Electronic temperature and density of the plasma produced by nanosecond ultraviolet laser ablation of LiF Appl. Phys. Lett. 86, 181501 (2005); 10.1063/1.1922574

Overcritical density plasma diagnosis inside laser-produced craters

Appl. Phys. Lett. 79, 177 (2001); 10.1063/1.1381413

Electron density and temperature measurements in a laser produced carbon plasma J. Appl. Phys. 82, 2140 (1997); 10.1063/1.366276

Kinetic to thermal energy transfer and interpenetration in the collision of laser-produced plasmas Phys. Plasmas 4, 190 (1997); 10.1063/1.872132

\section{A|P $\left.\right|_{\text {Applied Physics }} ^{\text {Journal of }}$}

Journal of Applied Physics is pleased to announce André Anders as its new Editor-in-Chief 


\title{
A method to measure the electron temperature and density of a laser-produced plasma by Raman scattering
}

\author{
H. Jang, ${ }^{1,2}$ M. S. Hur, ${ }^{3}$ J. M. Lee, ${ }^{2}$ M. H. Cho, ${ }^{1}$ W. Namkung, ${ }^{1}$ and H. Suk ${ }^{2, a)}$ \\ ${ }^{1}$ Department of Physics, Pohang University of Science and Technology, Pohang 790-784, Republic of Korea \\ ${ }^{2}$ APRI and School of Photon Science and Technology, Gwangju Institute of Science and Technology, \\ Gwangju 500-712, Republic of Korea \\ ${ }^{3}$ Korea Electrotechnology Research Institute, Ansan 426-170, Republic of Korea
}

(Received 16 April 2008; accepted 30 July 2008; published online 20 August 2008)

\begin{abstract}
A method is proposed to investigate the electron temperature and density of a laser-produced plasma simultaneously, using the temperature dependence difference of the Raman forward scattering (RFS) and backward scattering (RBS). Density and temperature dependence of spectra from the RBS and the RFS in a laser produced plasma were investigated by one-dimensional particle-in-cell simulations in the nonrelativistic regime. This technique has a great advantage as a simple diagnostic of plasma characteristics in the sense that it can be performed only with the pump laser, without any additional probe laser. (C) 2008 American Institute of Physics.
\end{abstract}

[DOI: $10.1063 / 1.2973395]$

A laser-produced plasma with a gas target has been studied extensively and intensively because of its wide applications for plasma-based particle acceleration, ${ }^{1,2}$ highbrightness X-ray generation, ${ }^{3}$ laser pulse amplification, ${ }^{4,5}$ attosecond x-ray generation, ${ }^{6}$ etc. Generally the physical properties of all those systems are strongly dependent on the status of the plasma. So it is very important to have proper diagnostics of the plasma for efficient experimental studies of the laser-plasma. There are several techniques to diagnose the plasma such as Thomson scattering, Raman spectroscopy, interferometry, etc. The Thomson scattering is widely used to obtain the plasma temperature and its temporal evolution at a well-defined position. ${ }^{7,8}$ In this method, a probe laser pulse should be used separately from a driving pump laser. The Raman spectroscopy from Raman forward scattering (RFS) is also a very convenient tool to get the plasma density without using a separate diagnostic pulse, in case of laser-produced plasma with a gas target. ${ }^{9}$ The interferometry technique is also available for the density measurement, and it can give us spatially resolved snapshots of the density. ${ }^{10}$ However, those two methods do not give us plasma temperature information, which is very important for plasma studies. In addition to the above methods, the Raman backscattering (RBS) can be used to measure the electron temperature ${ }^{11}$ of plasmas. In their experiments, they had to measure the electron density with the interferometry, since their method of temperature analysis required the electron density of the target plasma. Thus, those previously used techniques cannot give the plasma density and temperature simultaneously without using additional probe tools.

In this paper, we propose a method to simultaneously measure the time- and space-averaged plasma density and temperature by a single shot of a driving pump laser without using an additional probe. The parameters are averaged over a distance of the Reyleigh range for a focused laser beam. The idea is using the different behavior of RBS and RFS as a function of the plasma temperature. For RBS, the plasma wave is driven by the beat of two counterpropagating elec-

${ }^{a)}$ Electronic mail: hysuk@ gist.ac.kr. tromagnetic waves: one is the pump wave and the other is a daughter wave that is scattered backward. Because of the counterpropagating nature, the beat wavelength of the driving ponderomotive force is even shorter than the laser wavelength, which gives a large value of the Langmuir plasma wavenumber $\left(k_{L}\right)$, i.e., a slow wave. In this case, the dispersion relation of the Langmuir plasma wave is given by

$$
\omega_{L}^{2}=\omega_{p}^{2}+\frac{3}{2} v_{\mathrm{th}}^{2} k_{L}^{2}
$$

where $v_{\text {th }}$ is the thermal velocity of electrons, the huge number $k_{L}$ gives considerable thermal effects even for a nonrelativistic temperature $\left(v_{\mathrm{th}} / c \ll 1\right)$. On the contrary, for RFS, a fast plasma wave is driven, where $k_{L}$ has only a small value compared to the laser wavenumber. Thus the thermal effect in RFS is generally negligible for nonrelativistic plasma temperature.

In the regime of underdense plasmas, the incident electromagnetic wave is scattered into daughter electromagnetic waves and an electron plasma wave. For RFS, the strong electromagnetic pump wave $\left(\omega_{0}, k_{0}\right)$ decays into a plasma wave $\left(\omega_{p}, k_{p}\right)$ and two forward propagating electromagnetic waves with two frequencies, the Stokes $\left(\omega_{0}-\omega_{p}\right)$ and antiStokes $\left(\omega_{0}+\omega_{p}\right)$. For RBS, just the Stokes component is prone to the instability mechanism, leading to an exponential growth to an observable amplitude. ${ }^{12}$ The scattered light and the plasma wave obey the energy and momentum conservation as $\omega_{L}=\omega_{0}-\omega_{s}, \boldsymbol{k}_{L}=\boldsymbol{k}_{0}-\boldsymbol{k}_{s}$, where $\omega_{L}$ is the frequency of the Langmuir wave, $\omega_{s}$ is the scattered light frequency, $\boldsymbol{k}_{L}$ is the wavevector of the Langmuir wave, $\boldsymbol{k}_{0}$ is the wavevector of the incident laser, and $\boldsymbol{k}_{s}$ is the wavevector or the scattered light. The relation between the wavevectors is derived from the energy and momentum conservation as

$$
\left|\boldsymbol{k}_{L}\right|^{2}=\left|\boldsymbol{k}_{0}-\boldsymbol{k}_{s}\right|^{2}=k_{0}^{2}+k_{s}^{2}-2 k_{0} k_{s} \cos \theta,
$$

where $\theta$ is the angle between $k_{0}$ and $k_{s}$. If we assume $k_{s}$ $\simeq k_{0}$ for underdense plasmas $\left(\omega_{p} \ll \omega_{0, s}\right)$, 


$$
\left|\boldsymbol{k}_{L}\right|^{2}=2 k_{0}^{2}-2 k_{0}^{2} \cos \theta=2 k_{0}^{2}(1-\cos \theta) .
$$

In case of the RBS, $\theta \simeq \pi$ and $\left|\boldsymbol{k}_{L}\right|^{2} \simeq 4 k_{0}^{2}$. Hence, from Eq. (1), the thermal term relative to the cold plasma frequency becomes $1.5 \beta_{\mathrm{th}}^{2} c^{2} k_{L}^{2} / \omega_{p}^{2} \simeq 6\left(\beta_{\mathrm{th}} \omega_{0} / \omega_{p}\right)^{2}$, where $\beta_{\mathrm{th}}=v_{\mathrm{th}} / c$. Even for a nonrelativistic thermal velocity $\left(\beta_{\mathrm{th}} \ll 1\right)$, the underdense condition of the plasma $\left(\omega_{0} \gg \omega_{p}\right)$, together with the factor 6, gives a non-negligible thermal effect for RBS. In the RFS case, however, $\theta \simeq 0$ and $\left|\boldsymbol{k}_{L} / k_{0}\right|^{2} \ll 1$, which consequently leads to the strong independence of RFS on $v_{\text {th }}$.

To obtain the thermal velocity and temperature of the plasma from the wavenumber of the Raman backscattered signal, the above relations, the dispersion relations of the pump and scattered lights, which are given as $\omega_{0}^{2}=\omega_{p}^{2}+c^{2} k_{0}^{2}$, $\omega_{s}^{2}=\omega_{p}^{2}+c^{2} k_{s}^{2}$, and the condition $\theta=\pi$ (backscatter) in Eq. (2), are combined to yield the following relation between $k_{s}$ and $v_{\mathrm{th}}$ :

$$
\begin{gathered}
1-k_{s} \sqrt{\left(\frac{c}{\omega_{s}}\right)^{2}+\left(\frac{\omega_{p}}{\omega_{0} k_{s}}\right)^{2}+\frac{3}{2}\left(\frac{v_{\text {th }}}{\omega_{0}}\right)^{2}} \\
=\frac{\omega_{p}}{\omega_{0}} \sqrt{1+\frac{3}{2}\left(\frac{v_{\text {th }}}{\omega_{p}}\right)^{2}+\left(k_{s}+k_{0}\right)^{2}}
\end{gathered}
$$

for RBS, where $k_{s}$ is the wavenumber of the Raman backscattered signal. As mentioned above, the frequency of the Langmuir wave driven by the beat of Raman forward scattered signal and the pump wave is not influenced much by the thermal effects. Neglecting the thermal term in Eq. (1) yields

$$
\omega_{p}=\frac{\omega_{0}^{2}-c^{2} k_{s}^{2}}{2 \omega_{0}}
$$

for RFS, where $k_{s}$ is the wavenumber of the Stokes from the RFS. Equation (5) gives the plasma density for a given frequency of the driving pump laser.

In the laser plasma with a gas target, the idea can be applied as follows. The RFS and RBS signals from the driving pump laser are acquired simultaneously. Then the Stokes or anti-Stokes from RFS is used to determine the plasma density using Eq. (5). The additional shift of the RBS peak from the anti-Stokes location can be used to estimate the plasma temperature by Eq. (4). Note that in the previous experiments, only one of the RBS or RFS signals were captured to measure only one of the plasma temperature or density.

Though the scheme proposed here is eventually for experiments, the idea was tested by numerical simulations using the one-dimensional particle-in-cell code XOOPIC. ${ }^{13}$ In the simulations, the Stimulated Raman Scattering (SRS) spectra were obtained for varying $T_{e}$. We loaded a preformed plasma at the laser target position. The plasma frequency for the simulations was $\omega_{p} / \omega_{0}=0.132$, which corresponds to $n_{e}$ $=1.95 \times 10^{19} \mathrm{~cm}^{-3}$, and its temperature was varied from 0 to $300 \mathrm{eV}$. Note that the temperature range is in the nonrelativistic regime, which is commonly observed in the laser wakefield acceleration. The wavelength of the driving laser pulse is $1 \mu \mathrm{m}$ and the longitudinal duration is $700 \mathrm{fs}$. This pulse length is proper for the electron acceleration in the selfmodulated laser wakefield accelerator regime. The normalized vector potential, which is defined by $a=e A / m c^{2}$, is 0.2 . Here $A$ is the magnetic vector potential of the laser pulse, $m$ and $e$ are the unit charge and mass of the electron, respec-
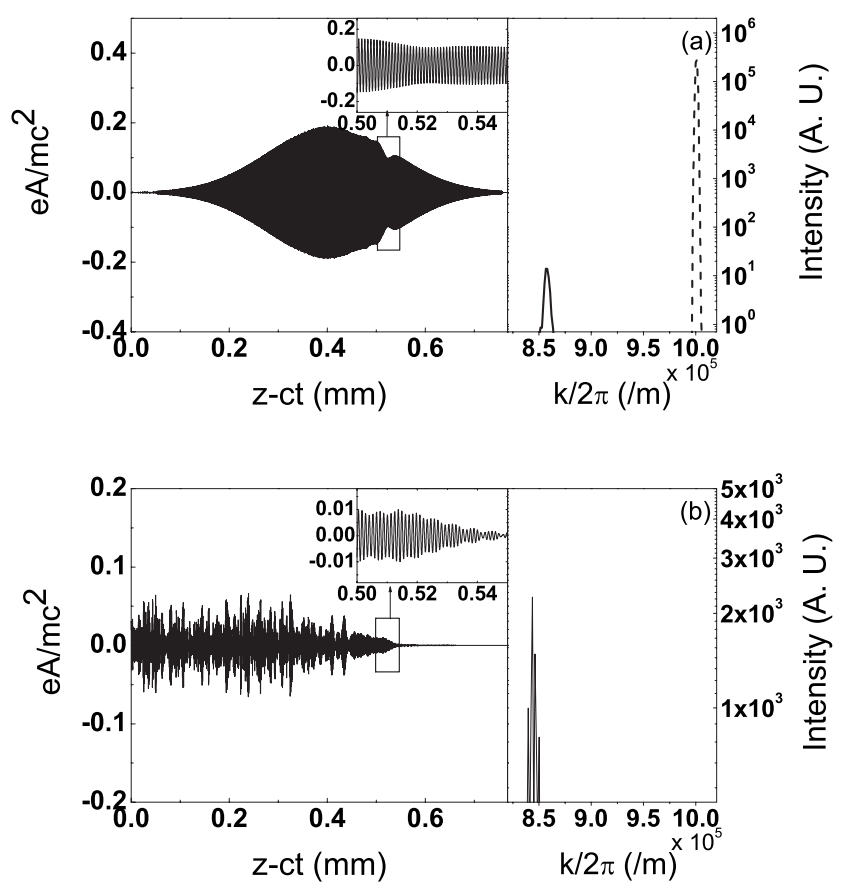

FIG. 1. Measured electric field data and their transformed spectra for $t=5.15 \mathrm{ps}$ and $T_{e}=200 \mathrm{eV}$. (a) is the right-propagating case (laser direction) and (b) is the left-propagating case (opposite to the laser direction). The dashed line in (a) is the spectrum of the incident laser pulse (unshifted main pulse).

tively, and $c$ is the speed of light in vacuum. A moving window technique was used to reduce the simulation time. The driving pump laser pulse propagates to the right direction in the simulation window.

The spectra of the pump and scattered lights were obtained by Fourier transformation of the electric and magnetic field data. To distinguish the backward and forward scattered spectra, the electromagnetic wave was separated: one propagates along the pump laser and the other propagates in the opposite direction by using the equation $E_{\mathrm{LD}}=\left(E_{y}-c B_{z}\right) / 2$, $E_{\mathrm{OD}}=\left(E_{y}+c B_{z}\right) / 2$, where $E_{\mathrm{LD}}$ is the electomagnetic wave which goes to the pump laser direction and $E_{\mathrm{OD}}$ is the electromagnetic wave which goes to the opposite direction. ${ }^{14} \mathrm{By}$ the fast Fourier transform on this electric field data, we could get the spectra of the backward and forward scattered lights. Figure 1 is the measured electric field data and their transformed spectrum for $t=5.15 \mathrm{ps}$ and $T_{e}=200 \mathrm{eV}$. Figure 1(a) shows the right-propagating case (laser direction) and Fig. 1(b) shows the left-propagating case (opposite to the laser direction). We compared these two SRS spectra to measure $n_{e}$ and $T_{e}$. Figure 2 shows the (a) RFS and (b) RBS spectrum in case of $T_{e}=0 \mathrm{eV}$ and $T_{e}=200 \mathrm{eV}$. These spectra were measured after the laser pulse propagated through the plasma by $770 \mu \mathrm{m}$. The peak location of RFS is almost fixed while the peak location of RBS moved considerably. Also the peak location of RFS agrees well with the expected peak location from Eq. (5) $\left(\omega_{s}=0.858 \omega_{0}\right.$ for $\left.\omega_{p} / \omega_{0}=0.132\right)$. From this result, we could confirm that the peak location of RBS light is affected significantly by $T_{e}$, while the peak location of the RFS light is not. To observe more peak location data with varying $T_{e}$, we launched several simulations with different electron temperatures.

Figure 3 is the summary of the simulation results. It shows the peak shift due to the change of $T_{e}$ for $t=5.45 \mathrm{ps}$. 

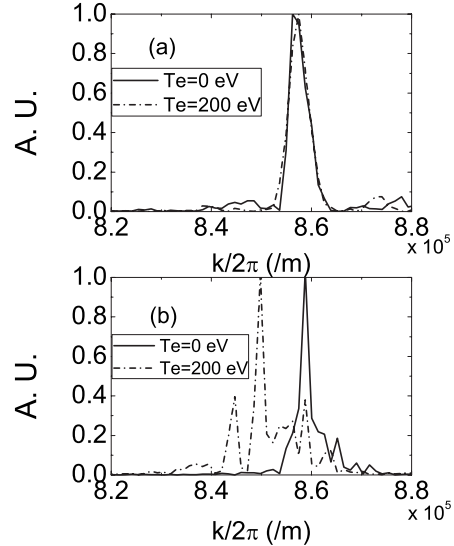

FIG. 2. (a) RFS and (b) RBS spectra in case of $T_{e}=0 \mathrm{eV}$ and $T_{e}=200 \mathrm{eV}$. Peak locations of the RFS signal are almost the same, but the peak locations of RBS signal show a big difference as $T_{e}$ is different.

The lines represent theoretically calculated locations, which are the solutions of Eqs. (4) and (5). Since we used a laser pulse whose a is 0.2, Eqs. (1) and (4) are valid without the relativistic treatment. For higher power application, the relativistic effect for the temperature should be considered. The circles (RBS) and the dots (RFS) are the peak locations of the scattered light measured from the simulations, which shows a good agreement with the theoretical values. As $T_{e}$ increases, the wavevector of the scattered light decreases and

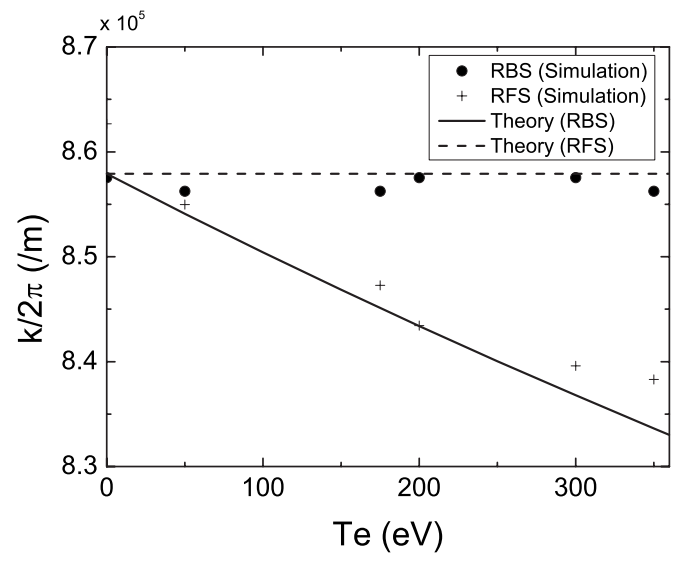

FIG. 3. The peak shift due to the change of $T_{e}$ for $t=5.45 \mathrm{ps}$. The lines are theoretically calculated locations of the RBS light, the circles are the peak locations of the RBS light, and the dots are the peak locations of the RFS light it is caused by the frequency change of the RBS light. $\omega_{L}$ is dependant on $T_{e}$ as mentioned in Eq. (1) for RBS and it affects the frequency and wavevector of the scattered light.

In conclusion, we proposed and studied a method utilizing the different sensitivity of RBS and RFS to the thermal velocity to simultaneously measure the electron temperature and density of a laser-produced plasma. For the studies, we conducted one-dimensional numerical simulations and the results showed a good agreement with the linear theory. The idea can be realized in experiments by measuring the RFS and RBS spectra in a single shot of a pump laser without using separate diagnostic tools such as a probe pulse or interferometry. This implies that the plasma diagnostics in the laser-plasma experiments can be greatly simplified and this method can be used as a tool for plasma diagnostics.

This work was financially supported by the Industrial Technology Infrastructure Building Program, Ministry of Knowledge Economy of Korea, and it was also supported by GIST Top Brand Project "Photonics 2020," Ministry of Education, Science and Technology of Korea.

${ }^{1}$ T. Tajima and J. M. Dawson, Phys. Rev. Lett. 43, 267 (1979).

${ }^{2}$ J. Faure, C. Rechatin, A. Norlin, A. Lifschitz, Y. Glinec, and V. Malka, Nature (London) 444, 737 (2006).

${ }^{3}$ D. L. Matthews, P. L. Hagelstein, M. D. Rosen, M. J. Eckart, N. M. Ceglio, A. U. Hazi, H. Medecki, B. J. MacGowan, J. E. Trebes, B. L. Whitten, E. M. Campbell, C. W. Hatcher, A. M. Hawryluk, R. L. Kauffman, L. D. Pleasance, G. Rambach, J. H. Scofield, G. Stone, and T. A. Weaver, Phys. Rev. Lett. 54, 110 (1985).

${ }^{4}$ G. Shvets, N. J. Fisch, A. Pukhov, and J. Meyer-ter-Vehn, Phys. Rev. Lett. 81, 4879 (1998).

${ }^{5}$ M. S. Hur, J. Kim, D. N. Gupta, H. J. Jang, and H. Suk, Appl. Phys. Lett. 91, 101501 (2007).

${ }^{6}$ P. B. Corkum, Phys. Rev. Lett. 71, 1994 (1993)

${ }^{7}$ A. Ting, K. Krushelnick, C. I. Moore, H. R. Burris, E. Esarey, J. Krall, and P. Sprangle, Phys. Rev. Lett. 77, 5377 (1996).

${ }^{8}$ C. Joshi, Phys. Plasmas 14, 055501 (2007).

${ }^{9}$ N. Hafz, M. S. Hur, G. H. Kim, C. Kim, I. S. Ko, and H. Suk, Phys. Rev. E 73, 016405 (2006).

${ }^{10}$ L. A. Gizzi, M. Galimberti, A. Giulietti, D. Giulietti, P. Koester, L. Labate, P. Tomassini, Ph. Martin, T. Ceccotti, P. De Oliveira, and P. Monot, Phys. Rev. E 74, 036403 (2006).

${ }^{11}$ C. L. Shepard, J. A. Tarvin, R. L. Berger, Gar. E. Busch, R. R. Johnson, and R. J. Schroeder, Phys. Fluids 29, 583 (1986).

${ }^{12}$ W. L. Kruer, J. M. Dawdon, and R. N. Sudan, Phys. Rev. Lett. 23, 838 (1969).

${ }^{13}$ J. P. Verboncoeur, A. B. Langdon, and N. T. Gladd, Comput. Phys. Commun. 87, 199 (1995).

${ }^{14}$ P. Mardahl, H. J. Lee, G. Penn, J. S. Wurtele, and N. J. Fisch, Phys. Lett. A 296, 109 (2002). 